\title{
A REPRESENTATION LATTICE ISOMORPHISM FOR THE PERIPHERICAL SPECTRUM
}

\author{
JOSEP MARTÍNEZ
}

(Communicated by Palle E. T. Jorgensen)

\begin{abstract}
In this paper we construct a representation isometric lattice isomorphism for the peripherical spectrum of a positive operator on a Banach lattice. By a representation lattice homomorphism, we mean that the peripherical spectrum of the operator is identified with the spectrum of the induced isometric lattice homomorphism. A simple proof of a "zero-two" law follows easily from our representation technique.
\end{abstract}

\section{THE REPRESENTATION LATTICE HOMOMORPHISM}

We develop our technique in the context of Banach lattices with $p$-additive norm. Following Zaanen [Zaa],

1.1. Definition. Let $1 \leq p<\infty$. A Banach lattice $E$ for which $\|x+y\|^{p}=$ $\|x\|^{p}+\|y\|^{p}$ whenever $x \wedge y=0$ is called an abstract $\mathscr{L}^{p}$-space (or an $A L_{p^{-}}$ space).

In fact, the norm of an $A L_{p}$-space $(1 \leq p<\infty)$ is $p$-superadditive for all positive elements. In other words, if $E$ is an $A L_{p}$-space, then

$$
\|x+y\|^{p} \geq\|x\|^{p}+\|y\|^{p} \quad \forall x, y \geq 0 .
$$

As a straightforward consequence of the $p$-superadditivity of the norm, we get the following basic property of $A L_{p}$-spaces:

(1) If $T$ is a contraction on $E$ that verifies $0 \leq x \leq T x$ for some $x \in E$, then $T x=x$.

Now let $E$ be an $A L_{p}$-space $(1 \leq p<\infty)$, and let $\mathscr{F}$ denote a free ultrafilter on $\mathbf{N}$. The $\mathscr{F}$-product $\widehat{E}_{\mathscr{F}}$ is actually an $A L_{p}$-space (see [S1, Chapter $\left.\mathrm{V}, \S 1\right]$ ). We denote by $P$ the following isometric lattice isomorphism on $\widehat{E}_{\mathscr{F}}$ :

$$
P\left(\left(x_{1}, x_{2}, \ldots\right)+c_{\mathscr{F}}(E)\right)=\left(x_{2}, x_{3}, \ldots\right)+c_{\mathscr{F}}(E),
$$

whose inverse isometric lattice homomorphism is given by

$$
Q\left(\left(x_{1}, x_{2}, \ldots\right)+c_{\mathscr{F}}(E)\right)=\left(0, x_{1}, x_{2}, \ldots\right)+c_{\mathscr{F}}(E) .
$$

Received by the editors February 13, 1992.

1991 Mathematics Subject Classification. Primary 47B55.

The author was supported by an F.P.I. grant from the Spanish Ministerio de Educación y Ciencia. 
1.2. Definition. Let $T$ be a positive contraction on $E$, and denote by $\widehat{T}_{\mathscr{F}}$ its canonical extension to the $\mathscr{F}$-product $\widehat{E}_{\mathscr{F}}$. We define the limit space of $T$ as the Banach space $E(T)=\operatorname{Ker}\left(P-\widehat{T}_{\mathscr{F}}\right)$ and the limit operator of $T$ by $\widetilde{T}=\left.\widehat{T}_{\mathscr{F}}\right|_{E(T)}=\left.P\right|_{E(T)}$. The fact that

$$
\left\{T^{n} x\right\}+c_{\mathscr{F}}(E) \in E(T) \quad \forall x \in E
$$

justifies our terminology. The Banach subspace

$$
A(T)=\overline{\left\{\left\{T^{n} x\right\}+c_{\mathscr{F}}(E): x \in E\right\}} \subseteq E(T)
$$

will be called the asymptotic space of $T$.

As an immediate consequence of property $(1), E(T)=\operatorname{Ker}\left(I-Q \widehat{T}_{\mathscr{F}}\right)$ is in fact a sublattice of $\widehat{E}_{\mathscr{F}}$ and so an $A L_{p}$-space. Moreover, as $\widetilde{T}$ is the restriction of an isometric lattice homomorphism, $\widetilde{T}$ is obviously an isometric lattice homomorphism.

We can now state our basic lemma, which translates the techniques of AllanRansford [A] and Phong-Lyubich [L] to the setting of Banach lattices.

1.3. Theorem. Let $T$ be a positive contraction on the $A L_{p}$-space $E$ with $1 \leq$ $p<\infty$, and let $\widetilde{T}$ denote its limit operator. Then we have

$$
\Gamma \cap \sigma_{p}(T) \subseteq \sigma(\widetilde{T}) \subseteq \Gamma \cap \sigma(T), \quad \text { where } \Gamma=\{\lambda \in \mathbf{C}:|\lambda|=1\} .
$$

Proof. Let $\lambda \in \rho(T)$. As

$$
R(\widehat{\lambda, T})(E(T)) \subseteq E(T),
$$

we get $\lambda \in \rho(\widetilde{T})$, where the resolvent is given by

$$
R(\lambda, \widetilde{T})=\left.R(\widehat{\lambda, T})\right|_{E(T)}
$$

But $\widetilde{T}$ is an invertible isometry and so the inclusion $\sigma(\widetilde{T}) \subseteq \Gamma \cap \sigma(T)$ is already proved.

On the other hand, if $\lambda \in \Gamma \cap \sigma_{p}(T)$, there exists a nonzero vector $x$ with $T x=\lambda x$. Defining now

$$
\tilde{x}=\left(x, \lambda x, \lambda^{2} x, \ldots\right)+c_{\mathscr{F}}(E) \in E(T),
$$

we obtain $\tilde{T} \tilde{x}=\lambda \tilde{x},\|\tilde{x}\|=\|x\| \neq 0$; therefore, $\lambda \in \sigma(\widetilde{T})$.

\section{APPLICATIONS}

Let $T$ be a positive contraction on $L^{1}$. In 1970 Orstein and Sucheston [O] showed that

$$
\sup _{\|f\|_{1} \leq 1} \lim _{n \rightarrow \infty}\left\|T^{n} f-T^{n+1} f\right\|_{1}
$$

is either 0 or 2. This surprising result opened a new direction of research. Wittmann [W] extended this "zero-two" law to $A L_{p}$-spaces. On the other hand, Zaharopol [Zah], Katznelson-Tzafriri [K], and Schaefer [S2] proved that, given a positive linear contraction in an arbitrary Banach lattice, the limit $\lim _{n}\left\|\left|T^{n}-T^{n+1} \|\right|\right.$ is either 0 or 2 .

We now deduce a simple proof of a uniform "zero-two" law from the above representation technique. We need the following modification of an ArendtSchaefer-Wolff result (see [Ar, Lemma 3.3]): 
2.1. Lemma. Let $T$ be a positive isometry on the Banach lattice $E$, and suppose that $r(I-T)<\sqrt{3}$. Then we have $T=I$.

Proof. By the classical result of Gelfand (see [A]), we only need to show $\sigma(T)=$ 1 . If this is not verified, as $\sigma(T)$ is cyclic (see [S1]), there must be an element $a \in \sigma(T)$ such that $\frac{2}{3} \pi \leq \arg a \leq \frac{4}{3} \pi$ holds; this implies $-1 \leq \operatorname{Re}(a) \leq-\frac{1}{2}$. From this inequality we obtain

$$
r(I-T)^{2} \geq|a-1|^{2}=|a|^{2}-2 \operatorname{Re}(a)+1 \geq 3 .
$$

2.2. Theorem. Let $E$ be an $A L_{p}$-space $(1 \leq p<\infty)$, and let $T$ be a positive contraction on $E$. Then the following statements are equivalent:

(a) $\lim _{n \rightarrow \infty}\left\|T^{n}-T^{n+1}\right\|=0$.

(b) $\lim _{n \rightarrow \infty}\left\|T^{n}-T^{n+1}\right\|<\sqrt{3}$.

Proof. (b) $\rightarrow$ (a) Given a free ultrafilter $\mathscr{F}$ on $E$, if we denote by $S=\widehat{T}_{\mathscr{F}}$ the canonical extension of $T$ to the $\mathscr{F}$-product $A L_{p}$-space $\widehat{E}_{\mathscr{F}}$, we have (see [S1, Chapter V, §1])

$$
\sigma_{a p}(T)=\sigma_{p}(S), \quad\left\|T^{n}-T^{n+1}\right\|=\left\|S^{n}-S^{n+1}\right\| .
$$

By the Katznelson-Tzafriri theorem [K], it suffices to prove $\sigma_{p}(S) \cap \Gamma=\sigma(T) \cap$ $\Gamma \subseteq\{1\}$. However, given $x \in E(T)$, denoting by $\widetilde{S}$ the limit operator of $S$ we get

$$
\|x-\tilde{S} x\|=\left\|Q^{n}\left(\widehat{S}^{n}-\widehat{S}^{n+1}\right) x\right\| \leq\left\|S^{n}-S^{n+1}\right\|\|x\|
$$

and so we deduce $\|I-\widetilde{S}\|<\sqrt{3}$. Lemma 2.1 now shows that $\widetilde{S}=I$, and then from Theorem 1.3 we conclude

$$
\Gamma \cap \sigma_{p}(S) \subseteq \sigma(\widetilde{S})=\{1\} .
$$

\section{ACKNOWLEDGMENT}

The author wishes to thank Professors F. Andreu and J. Mazón for many stimulating conversations concerning the subject of this paper.

\section{REFERENCES}

[A] G. R. Allan and T. J. Ransford, Power-dominated elements in Banach algebras, Studia Math. 94 (1989), 63-79.

[Ar] W. Arendt, H. H. Schaefer, and M. Wolff, On lattice isomorphisms with positive real spectrum and groups of positive operators, Math. Z. 164 (1978), 115-123.

[K] Y. Katznelson and C. Tzafriri, On power bounded operators, J. Funct. Anal. 68 (1986), 313-328.

[L] Y. I. Lyubich and Vu Quoc Phong, Asymptotic stability of linear differential equations in Banach spaces, Studia Math. 88 (1988), 37-42.

[O] D. Orstein and L. Sucheston, An operator theorem on $L^{1}$ convergence to zero with applications to Markov kernels, Ann. Math. Statist. 41 (1970), 1631-1639.

[S1] H. H. Schaefer, Banach lattices and positive operators, Springer, Berlin, Heidelberg, and New York, 1974.

[S2] _ The zero-two law for positive contractions is valid in all Banach lattices, Israel J. Math. 59 (1987), 241-244.

[W] R. Wittmann, Analogues of the zero-two law for positive linear contractions in $L^{p}$ and $C(X)$, Israel J. Math. 59 (1987), 8-28. 
[Zaa] A. C. Zaanen, Riesz spaces. II, North-Holland, New York, 1983.

[Zah] R. Zaharopol, The modulus of a regular linear operator and the "zero-two" law in $L^{p}$-spaces $(1<p<\infty, p \neq 2)$, J. Funct. Anal. 68 (1986), 300-312.

Departament d'Analisi Matemàtica, Universitat de Valencia, Dr. Moliner 50, 46100 BURJASSOT, SPAIN

E-mail address: martinjo@vm.ci.uv.es 